\title{
Direct Measurement of the Dissipation Rate Spectrum around Ion Kinetic Scales in Space Plasma Turbulence
}

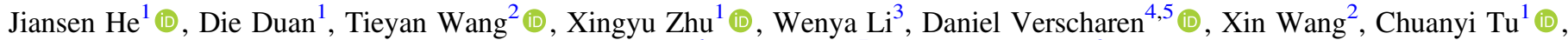 \\ Yuri Khotyaintsev ${ }^{6}$ (10), Guan $\mathrm{Le}^{7}$ (10), and Jim Burch ${ }^{8}$ \\ ${ }^{1}$ School of Earth and Space Sciences, Peking University, Beijing 100871, Beijing, People's Republic of China; jshept@pku.edu.cn \\ ${ }^{2}$ School of Space and Environment, Beihang University, Beijing 100083, People's Republic of China \\ ${ }^{3}$ State Key Laboratory of Space Weather, National Space Science Center, Chinese Academy of Sciences, Beijing 100190, People's Republic of China \\ ${ }^{4}$ Mullard Space Science Laboratory, University College London, Dorking RH5 6NT, UK \\ ${ }^{5}$ Space Science Center, University of New Hampshire, Durham, NH 03824, USA \\ ${ }^{6}$ Swedish Institute of Space Physics, Uppsala SE-75121, Sweden \\ ${ }^{7}$ NASA Goddard Space Flight Center, Greenbelt, MD 20771, USA \\ Southwest Research Institute, San Antonio, TX 78238, USA \\ Received 2019 January 2; revised 2019 May 15; accepted 2019 June 2; published 2019 August 1
}

\begin{abstract}
The energy of turbulence in the universe, which cascades from large fluid scales to small kinetic scales, is believed to be dissipated through conversion to thermal or nonthermal kinetic energy. However, identifying the dissipation processes and measuring the dissipation rate in turbulence remain challenging. Based on unprecedented highquality measurements of space plasma turbulence by the Magnetospheric Multiscale mission, we propose a novel approach to measure the scale-dependent spectrum of the energy conversion rate between the fluctuating electromagnetic energy and plasma kinetic energy. The energy conversion rate spectrum is found to show a positive bulge around the ion kinetic scale, which clearly indicates the dissipation of the turbulent energy. The energy dissipation rate around the ion scale is estimated to be $0.5 \times 10^{6} \mathrm{~J} \mathrm{~kg}^{-1} \mathrm{~s}^{-1}$. This work provides basic information on local dissipation in magnetosheath turbulence and sets up a new paradigm for studying the dissipation of universal plasma turbulence.
\end{abstract}

Key words: plasmas - turbulence - waves

\section{Introduction}

Turbulence is a prevalent phenomenon in various environments, playing a crucial role in the energy transfer across scales and energy conversion between turbulent energy and kinetic energy (Frisch 1995; Tu \& Marsch 1995; Biskamp 2003; Scannapieco \& Brüggen 2008; Bruno \& Carbone 2013; Burch et al. 2016; Hadid et al. 2018). When turbulence is in dynamic equilibrium, the energy cascade rate, dissipation rate, and heating rate are statistically the same (Howes 2015). However, the quantitative estimate of these three rates remains a challenging task. Turbulent cascade is caused by nonlinear interactions between eddies, waves, and structures. To describe the energy cascade in incompressible hydrodynamics, the Kolmogorov-Yaglom (KY's) law based on third-order structure functions was proposed (Kolmogorov 1941; Monin \& Yaglom 1975). For incompressible magnetohydrodynamic (MHD) turbulence, Elsässer variables were introduced, and an extension to KY's law was adopted as the Politano-Pouquet (PP's) law (Politano \& Pouquet 1998). To describe compressible MHD turbulence, a new law was derived by taking into account the contributions from compressible fluctuations (Banerjee \& Galtier 2013). The ensemble average of the signed cascade rate (Osman et al. 2011) in solar wind turbulence as derived from PP's law is estimated to be between $10^{3}$ and $10^{4} \mathrm{~J} \mathrm{~kg}^{-1} \mathrm{~s}^{-1}$, corresponding to $\left[10^{-16}, 10^{-15}\right]$ $\mathrm{J} \mathrm{m}^{-3} \mathrm{~s}^{-1}$ for an assumed proton number density of $6 \mathrm{~cm}^{-3}$. In the magnetosheath, the average of the absolute (unsigned) cascade rate is estimated to approach $10^{-13} \mathrm{~J} \mathrm{~m}^{-3} \mathrm{~s}^{-1}$ at its maximum level, at least two orders of magnitude larger than its counterpart in the solar wind (Hadid et al. 2018).
Our goal is to investigate how the energy is dissipated and how the dissipation rate is distributed over scales. This is one of the critical turbulence issues. In neutral gases, the viscosity due to molecular diffusion dissipates the turbulent energy at places of nonzero velocity gradients. In collisional ionized gases with a magnetic field, in addition to the viscosity, the resistivity due to collisions between particle species also contributes to the dissipation process. In collisionless plasmas, in which classical viscosity and resistivity are weak, turbulent electromagnetic fields at particle kinetic scales scatter the particles and reduce the current densities as well as transform the electromagnetic energy (Karimabadi et al. 2013). The wave-like turbulence at kinetic scales can be characterized as ion cyclotron, kinetic (inertial) Alfvén, or whistler-like in its dynamics, which introduces dissipation and nonlinear dispersion to deform the shape of the power spectral profile (Leamon et al. 1998; Bale et al. 2005; Gary \& Smith 2009; Narita et al. 2010; Sahraoui et al. 2010; He et al. 2011; Matteini et al. 2016; Chen \& Boldyrev 2017). On the other hand, increasing intermittency with locally enhanced current density sets in when moving from the MHD regime to the kinetic regime (Sorriso-Valvo et al. 1999; Kiyani et al. 2009). The intermittent structures are categorized as various types of discontinuities as well as the boundaries of pressurebalanced structures (Wang et al. 2013). Therefore, the dissipation of kinetic wave modes and the intermittent structures are regarded as the two major means of dissipation and responsible for plasma energization (Chandran et al. 2010; Wan et al. 2015). Both kinetic wave modes and intermittent structures owe their dissipation to energy transfer between fields and particles.

A main challenge and goal of space plasma investigations is the search for observational evidence for resonant interactions and energy conversion between plasma particles and turbulent 
waves. Some pieces of observational evidence can be used as indirect evidence of wave damping and plasma heating. For example, the break in the turbulence power spectral profile beyond the inertial range is found at the scale representing the ion cyclotron resonance (Bruno \& Trenchi 2014; Duan et al. 2018; Wang et al. 2018; Woodham et al. 2018). The coexistence of two wave modes (quasi-parallel ion cyclotron waves (ICWs) and quasi-perpendicular kinetic Alfvén waves (KAWs)) and three resonance diffusion plateaus in proton velocity space indicates a complicated scenario of waveparticle interactions in solar wind turbulence: left-handed cyclotron resonance between ICWs and the proton core population, Landau and right-handed cyclotron resonances between KAWs and the proton beam population ( $\mathrm{He}$ et al. 2015).

It is critical to understand the means through which the particle velocity distribution gains energy from the fluctuating fields during the energy conversion processes. Ruan et al. (2016) illustrated good correlation between $\delta f$ (instant deviation from the background Maxwellian distribution) and $\delta E_{\|}$(more specifically, $\left.\mathrm{CC}\left(\delta f\left(v_{\|}>0\right), \delta E_{\|}\right)>0, \mathrm{CC}\left(\delta f\left(v_{\|}<0\right), \delta E_{\|}\right)<0\right)$ by employing their Vlasov tool to simulate the damping of slow magnetosonic waves in the solar corona. Recently, Howes and his colleagues have been working intensively to develop a fieldparticle correlation technique and applied this technique to various gyrokinetic model outputs (Klein \& Howes 2016; Klein et al. 2017; Howes et al. 2017, 2018). By utilizing the field-particle correlation technique, Chen et al. (2019) reported a successful measurement of secular energy transfer from $\delta E_{\|}$to electrons, suggesting a signature of Landau damping of KAWs.

The $\boldsymbol{J} \cdot \boldsymbol{E}$ term is often studied in observational time series and in simulation data to quantify the dissipation of magnetic energy associated with coherent structures, e.g., magnetic reconnection (Zenitani et al. 2011; Osman et al. 2015; Fu et al. 2017). Aside from the $\boldsymbol{J} \cdot \boldsymbol{E}$ term, the term for pressure-strain tensor interaction, $-\boldsymbol{P} \cdot \nabla \cdot \boldsymbol{V}$, is another proxy for energy dissipation, representing the energy conversion from bulk kinetic energy to thermal kinetic energy (Yang et al. 2017; Chasapis et al. 2018; Sitnov et al. 2018). Simulations suggest that the spatial patterns of $\boldsymbol{J} \cdot \boldsymbol{E}$ and $-\boldsymbol{P} \cdot \nabla \cdot \boldsymbol{V}$ are often concentrated in proximity to each other (Yang et al. 2019). However, it is still difficult to quantify the Joule-like dissipation as a function of scale in turbulence observations.

The magnetosheath, which is the compressed solar wind downstream of the bow shock, is comparable to many other cosmic plasmas, e.g., the sheath of an astrosphere, the interstellar medium, or the remnants of supernovae. Various types of plasma waves have been identified in the magnetosheath turbulence (Anderson et al. 1991; Gary et al. 1993). What the dissipation rate is in sheath turbulence is an important issue. The newly implemented Magnetospheric Multiscale (MMS) mission, one of the most advanced spacecraft constellations dedicated to in situ measurement of space plasmas, provides unprecedented highresolution data of particles and fields. Measurements of 3D electric and magnetic fields simultaneously by four spacecraft with a separation distance of order $10 \mathrm{~km}$, make it now possible to study the dissipation rate spectrum of space plasma turbulence.

\section{Observations, Data Reduction, and Analysis Results}

\subsection{Turbulence Observation and Wave Identification}

As an example, we take the measurements of magnetosheath turbulence by $M M S$ during [08:00, 09:00] UT on 2015 October 3.
The plasma and field measurements are obtained from the Fast Plasma Investigation (FPI; Pollock et al. 2016) and FIELDS (Torbert et al. 2016) instruments, respectively. The $M M S X-Y$ position in GSE coordinates together with the 2D cuts of the predicted bow shock and magnetopause positions is plotted in Figure 1(a). In Figures 1(b)-(d), the general anticorrelation between magnetic field fluctuations $\left(B_{\mathrm{x}}, B_{\mathrm{y}}, B_{\mathrm{z}}\right)$ and velocity fluctuations $\left(V_{\mathrm{x}}, V_{\mathrm{y}}, V_{\mathrm{z}}\right)$ indicates the anti-Sunward propagation of Alfvénic waves along the magnetic field direction. The electric field fluctuations displayed in Figure 1(e) are given by $\boldsymbol{E}_{\left\langle\boldsymbol{V}_{i}\right\rangle}=\boldsymbol{E}+\left\langle\boldsymbol{V}_{\text {ion }}\right\rangle \times \boldsymbol{B}$, which is calculated by removing the convection electric fields due to ion mean bulk flow $\left\langle\boldsymbol{V}_{\text {ion }}\right\rangle$ from the original $\boldsymbol{E}$. Fluctuations of $\boldsymbol{B}$ and $\boldsymbol{E}_{\left\langle\boldsymbol{V}_{i}\right\rangle}$ at shorter periods (i.e., of order $1 \mathrm{~s}$ ) can also be identified in Figures 1(b)-(e). They are highlighted by red rectangles in Figures 1(f)-(g). Furthermore, based on the singular value decomposition (SVD) of the electromagnetic spectral matrix according to Gauss's and Faraday's laws (Santolík et al. 2003), the fluctuations are found to be left-hand circularly polarized about the local mean magnetic field direction $\left(\boldsymbol{B}_{0, \text { local }}\right)$ and propagate parallel to $\boldsymbol{B}_{0, \text { local }}$ (Figures 1(h)(i)), strongly suggesting their nature as ion cyclotron waves. The proton gyroperiod is $\sim 1.4 \mathrm{~s}$.

\subsection{Formula for Calculating the Energy Conversion Rate Spectrum}

The electric field is dependent on the reference frame. For example, $\delta \boldsymbol{E}=\boldsymbol{E}+\langle\boldsymbol{V}\rangle \times \boldsymbol{B}$ and $\delta \boldsymbol{E}^{\prime}=\boldsymbol{E}+\boldsymbol{V} \times \boldsymbol{B}$ represent the electric fields in the mean and local bulk flow reference frames, respectively. Which form should be used to calculate the spectrum of the energy conversion rate: $\langle\delta \boldsymbol{J} \cdot \delta \boldsymbol{E}\rangle$ or $\left\langle\delta \boldsymbol{J} \cdot \delta \boldsymbol{E}^{\prime}\right\rangle$ ? We compare $\langle\delta \boldsymbol{J} \cdot \delta \boldsymbol{E}\rangle,\left\langle\delta \boldsymbol{J} \cdot \delta \boldsymbol{E}^{\prime}\right\rangle$, and $2 \gamma \cdot\left\langle\delta B^{2}\right\rangle / 2 \mu_{0}$ for Alfvén waves (MHD and kinetic regimes) in wave-vector space $\left(k_{\|}\right.$, $\left.k_{\perp}\right)$. As a result, it is found that $\langle\delta \boldsymbol{J} \cdot \delta \boldsymbol{E}\rangle$ rather than $\left\langle\delta \boldsymbol{J} \cdot \delta \boldsymbol{E}^{\prime}\right\rangle$ is consistent with $2 \gamma \cdot\left\langle\delta B^{2}\right\rangle / 2 \mu_{0}$ (see Figure 2 ).

Traditionally, the "frame-independent" dissipation measure, $\boldsymbol{J} \cdot \boldsymbol{E}^{\prime}$, is adopted to calculate the direct energy conversion rate from the electromagnetic energy $E_{\mathrm{EM}}$ to the particle kinetic energy $E_{\mathrm{k}}$, which is a sum of thermal and nonthermal parts. The symbol $\boldsymbol{E}^{\prime}$ represents the electric field in the electron or ion local bulk flow reference frame, a result of subtracting the convection electric field, $\left(-\boldsymbol{V}_{\mathrm{e}} \times \boldsymbol{B}\right)$ or $\left(-\boldsymbol{V}_{i} \times \boldsymbol{B}\right)$, from the original $\boldsymbol{E}$ in the "rest frame of reference." In this way, the direct work done on the fluid motion by the Lorentz force of the EM field, $(\boldsymbol{J} \times \boldsymbol{B}) \cdot V_{\mathrm{e}}$ or $(\boldsymbol{J} \times \boldsymbol{B}) \cdot \boldsymbol{V}_{i}$, is eliminated from the energy transfer rate. Moreover, we need to consider the secular energy transfer rate, the time and/or volume average of the $\boldsymbol{J} \cdot \boldsymbol{E}^{\prime}$ time variation and/or its spatial distribution, to account for the real dissipation rate rather than including the oscillatory energy transfer rate, which is significantly larger than the secular rate. However, the secular energy transfer rate from electromagnetic energy to thermal energy, $\left\langle\delta \boldsymbol{J} \cdot \delta \boldsymbol{E}^{\prime}\right\rangle$, does not cover the entire picture of the dissipation of turbulent EM fields. By comparing the spectra of $2 \gamma \cdot\left\langle\delta B^{2}\right\rangle / 2 \mu_{0}$ (Figure 2(a)), $\langle\delta \boldsymbol{J} \cdot \delta \boldsymbol{E}\rangle$ (Figure 2(b)), and $\left\langle\delta \boldsymbol{J} \cdot \delta \boldsymbol{E}^{\prime}\right\rangle$ (Figures 2(c) and (d)) in wavenumber space $\left(\mathrm{k}_{/ /}, k_{\perp}\right)$ according to linear Vlasov-Maxwell theory, we find that $\langle\delta \boldsymbol{J} \cdot \delta \boldsymbol{E}\rangle$ rather than $\left\langle\delta \boldsymbol{J} \cdot \delta \boldsymbol{E}^{\prime}\right\rangle$ agrees better with $2 \gamma \cdot\left\langle\delta B^{2}\right\rangle / 2 \mu_{0}$, and $\langle\delta \boldsymbol{J} \cdot \delta \boldsymbol{E}\rangle$ is significantly (one order of magnitude) greater than $\left\langle\delta \boldsymbol{J} \cdot \delta \boldsymbol{E}^{\prime}\right\rangle$ in the high- $k$ region. The relation between the three quantities is 

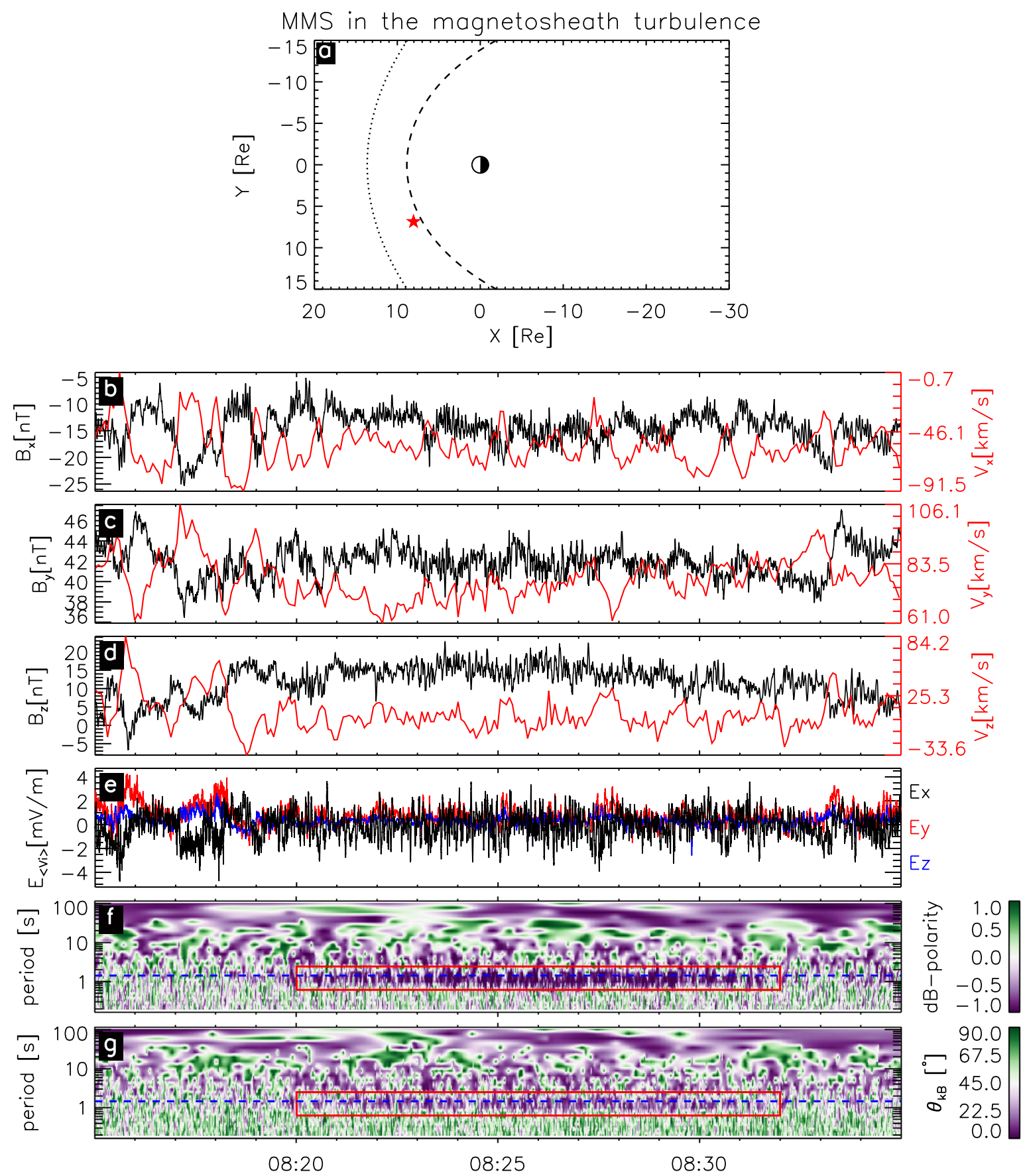

Figure 1. Alfvénic fluctuations and ion cyclotron waves in magnetosheath turbulence as measured by $M M S$ on 2015 October 3. (a) Position of the $M M S$ during the measurement projected onto the GSE $X-Y$ plane (red star). It is located between the bow shock (dotted curve) and magnetopause (dashed curve), both of which are predicted from experimental models with upstream solar wind data input. (b)-(d) Time sequence of magnetic field components $\left(B_{\mathrm{x}}, B_{\mathrm{y}}\right.$, and $\left.B_{\mathrm{z}}\right)$ and proton velocity components $\left(V_{\mathrm{x}}, V_{\mathrm{y}}\right.$, and $\left.V_{\mathrm{z}}\right)$ in GSE coordinates. The anticorrelation between the $B$-component and the $V$-component indicates an Earthward propagation of Alfvén waves. (e) Time sequence of the three components of $\boldsymbol{E}_{\langle V i\rangle}=\boldsymbol{E}+\left\langle\boldsymbol{V}_{i}\right\rangle \times \boldsymbol{B}$, the electric field in the proton mean bulk flow frame. (f) Sense of polarization for $d \boldsymbol{B}_{\perp}$ around the local mean magnetic field direction $\boldsymbol{B}_{0, \text { local }}$, with the values of -1 and +1 representing the left-hand and right-hand circular polarization about $\boldsymbol{B}_{0, \text { local }}$. (g) Angle of propagation direction for electromagnetic field fluctuations with respect to $\boldsymbol{B}_{0, \text { local }}, \boldsymbol{\theta}_{\mathrm{kB}}$. The existence of ICWs is highlighted with red rectangles in panels (f and $\mathrm{g}$ ), illustrating the characteristics of ICWs (left-hand polarization of $d \boldsymbol{B}_{\perp}, \boldsymbol{\theta}_{\mathrm{kB}} \sim 0^{\circ}$ ).

hence expressed as

$$
2 \gamma \cdot \frac{\left\langle\delta B^{2}\right\rangle}{2 \mu_{0}} \simeq\langle\delta \boldsymbol{J} \cdot \delta \boldsymbol{E}\rangle>\left\langle\delta \boldsymbol{J} \cdot \delta \boldsymbol{E}^{\prime}\right\rangle
$$

Because $\delta \boldsymbol{E}^{\prime}$ is in the non-inertial reference frame, one needs to consider the work done by inertial force in order to keep the energy conservation. The contribution from the work done by the inertial force to the energy conversion between fields and plasmas is not considered in $\left\langle\delta \boldsymbol{J} \cdot \delta \boldsymbol{E}^{\prime}\right\rangle$; therefore, the following inequality exists: $\left\langle\delta \boldsymbol{J} \cdot \delta \boldsymbol{E}^{\prime}\right\rangle\left\langle 2 \gamma \cdot \frac{\left\langle\delta B^{2}\right\rangle}{2 \mu_{0}}\right.$.

\subsection{Dynamic Spectrum of the Energy Conversion Rate}

Ion cyclotron resonance is an important dissipation mechanism in space plasmas. The oscillation of the $\boldsymbol{J} \cdot \boldsymbol{E}_{\left\langle\boldsymbol{V}_{i}\right\rangle}$ time 

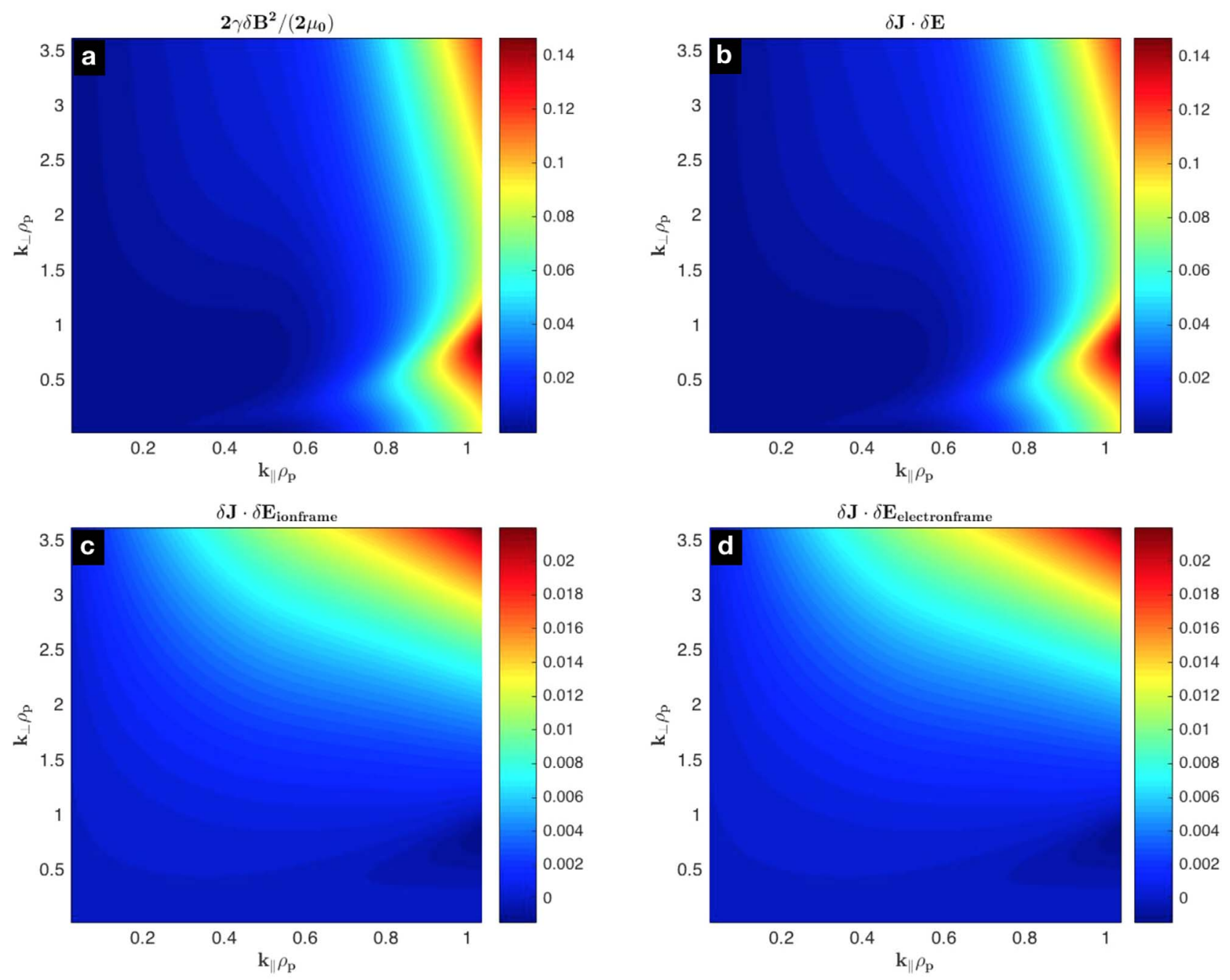

Figure 2. Feasibility demonstration of adopting $\langle\boldsymbol{\delta} \boldsymbol{J} \cdot \boldsymbol{\delta} \boldsymbol{E}\rangle$ to describe the damping rate spectrum $2 \gamma \cdot\left\langle\boldsymbol{\delta} \boldsymbol{B}^{2}\right\rangle / 2$. (a)-(d) Wavenumber space distributions of $2 \gamma|\delta \boldsymbol{B}|^{2} / 2 \mu_{0}$, $\frac{1}{4}\left(\delta \widetilde{\boldsymbol{J}} \cdot \delta \widetilde{\boldsymbol{E}}^{*}+\delta \widetilde{\boldsymbol{J}}^{*} \cdot \delta \widetilde{\boldsymbol{E}}\right), \frac{1}{4}\left(\delta \widetilde{\boldsymbol{J}} \cdot \delta \widetilde{\boldsymbol{E}}_{\text {IonFrame }}^{*}+\delta \widetilde{\boldsymbol{J}}^{*} \cdot \delta \widetilde{\boldsymbol{E}}_{\text {IonFrame }}\right)$, and $\frac{1}{4}\left(\delta \widetilde{\boldsymbol{J}} \cdot \delta \widetilde{\boldsymbol{E}}_{\text {ElectronFrame }}^{*}+\delta \widetilde{\boldsymbol{J}}^{*} \cdot \delta \widetilde{\boldsymbol{E}}_{\text {ElectronFrame }}\right)$, respectively. The label on top of each panel refers to the corresponding formula. Here, $\delta E^{\prime}$ for (c) and (d) represents $\delta \boldsymbol{E}_{\text {IonFrame }}=\delta E+\delta \boldsymbol{V}_{\text {ion }} \times \boldsymbol{B}_{0}$ and $\delta \boldsymbol{E}_{\text {ElectronFrame }}=\delta E+\delta \boldsymbol{V}_{\text {Electron }} \times \boldsymbol{B}_{0}$, respectively. Note that, for the sake of simplicity, $\delta B_{\perp 2}$ (magnetic field fluctuation in the second perpendicular direction out of the $\boldsymbol{k}-\boldsymbol{B}$ plane) is set to be the same in wavenumber space, which does not affect the comparison between different panels.

sequence around zero (Figure 3(a)) is not sufficient to provide insight into the essence of dissipation. To investigate whether the identified ion cyclotron waves are damped or growing, we calculate the spectrum of the energy conversion rate in units of $\mathrm{J} \mathrm{s}^{-1} \mathrm{~m}^{-3} \mathrm{~Hz}^{-1}$, which is defined as

$$
\varepsilon_{\mathrm{ECR}}=\frac{1}{4}\left(\delta \widetilde{\boldsymbol{J}} \cdot \delta \widetilde{\boldsymbol{E}}^{*}+\delta \widetilde{\boldsymbol{J}}^{*} \cdot \delta \widetilde{\boldsymbol{E}}\right),
$$

where $\delta \widetilde{\boldsymbol{J}}, \delta \widetilde{\boldsymbol{J}}^{*}, \delta \widetilde{\boldsymbol{E}}$, and $\delta \widetilde{\boldsymbol{E}}^{*}$ are the wavelet spectra of $\boldsymbol{J}$ and $\boldsymbol{E}_{\left\langle V_{i}\right\rangle}$ as well as their conjugate counterparts. The critical wavenumbers of proton kinetics $\left(k d_{\mathrm{p}} \sim 1\right.$ and $k \rho_{\mathrm{p}} \sim 1$, where $d_{\mathrm{p}}$ is the proton inertial length and $\rho_{\mathrm{p}}$ is the proton gyroradius) correspond to the frequencies of 0.4 and $0.7 \mathrm{~Hz}$ in the spacecraft reference frame during the observed time interval. Therefore, a period larger than $2 \mathrm{~s}$ can be considered as the MHD inertial range where protons are fully magnetized. The oscillation of $\varepsilon_{\mathrm{ECR}}$ between negative and positive values at periods larger than $2 \mathrm{~s}$ suggests the lack of any net energy conversion between turbulent electromagnetic energy and plasma kinetic energy (Figure 3(b)). This is as expected because the turbulent energy at MHD scales mainly cascades across scales rather than being converted to energize the plasmas. At scales between 0.6 and $2 \mathrm{~s}$, as highlighted with the red rectangle, $\varepsilon_{\mathrm{ECR}}$ no longer alternates symmetrically between negative and positive values but stays mainly positive (Figure 3(b)).

After dividing $-\varepsilon_{\mathrm{ECR}}$ by the electromagnetic energy, we obtain a local pseudo-damping rate in units of $1 \mathrm{~s}^{-1}$,

$$
\gamma=\frac{1}{2} \frac{-1 / 4\left(\widetilde{\boldsymbol{J}} \cdot \widetilde{\boldsymbol{E}}^{*}+\widetilde{\boldsymbol{J}}^{*} \cdot \widetilde{\boldsymbol{E}}\right)}{|\delta \widetilde{\boldsymbol{B}}|^{2} / 2 \mu_{0}+\varepsilon_{0}|\delta \widetilde{\boldsymbol{E}}|^{2} / 2} .
$$

The value of $\gamma$ at scales of $[0.6,2] \mathrm{s}$ is about $-0.5 \mathrm{~s}^{-1}$ (Figure 3(c)), corresponding to a lifetime on the order of seconds for the turbulent electromagnetic energy to be dissipated. This lifetime suggests that the damping of ion cyclotron waves is a major contributor to the dissipation in this case. Therefore, we acquire preliminary evidence for ion cyclotron wave damping in the magnetosheath turbulence at this time. 

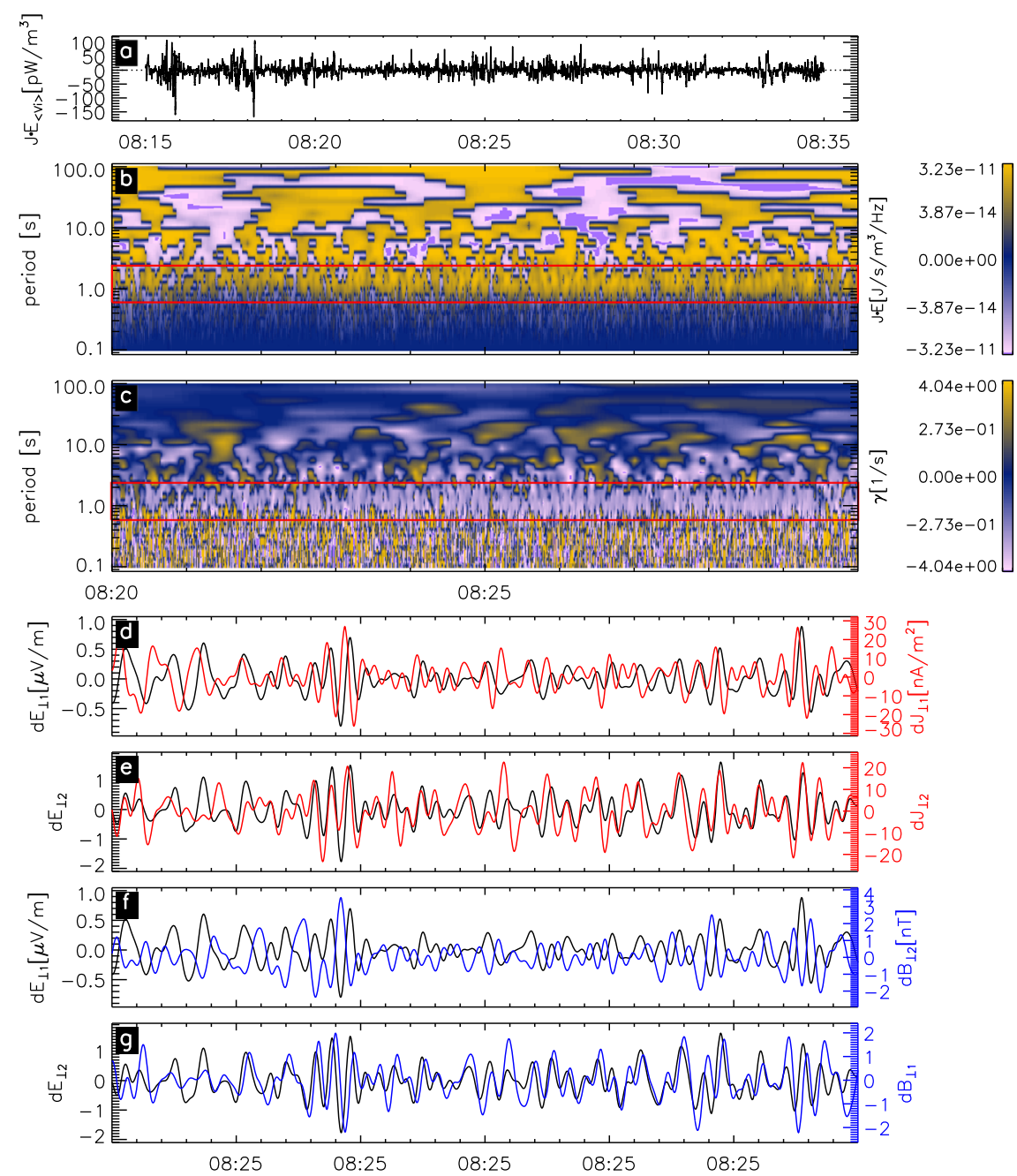

Figure 3. Direct evidence of dissipation of the magnetosheath turbulence around ion kinetic scales. (a) Oscillation of $\boldsymbol{J} \cdot \boldsymbol{E}_{\langle V i\rangle}$ around zero. (b) Time-period spectra of $\frac{1}{4}\left(\delta \widetilde{\boldsymbol{J}} \cdot \delta \widetilde{\boldsymbol{E}}^{*}+\delta \widetilde{\boldsymbol{J}}^{*} \cdot \delta \widetilde{\boldsymbol{E}}\right)$ showing the local pseudo-energy conversion rate spectra between electromagnetic and plasma energies, with the gold and desaturated blue colors denoting local positive and negative values, respectively. The time period of interest is highlighted with a red box, displaying a distribution with prevalent positive values. (c) Spectra of $\gamma$, with its mean value averaged over an interval lasting a couple of periods having a similar meaning to the growth/damping rate in plasma wave theory. The region with a prominent signature of net damping is also highlighted with a red box. (d) and (e) Subband of $\delta \boldsymbol{E}_{\perp}$ and $\delta \boldsymbol{J}_{\perp}$, as obtained from wavelet decomposition in the period range of $[0.6,3] \mathrm{s}$, showing clear coherence between one another, with phase lags generally smaller than $90^{\circ}$. The coherence with small phase lag causes $\left\langle\delta \boldsymbol{J}_{\perp}, \delta \boldsymbol{E}_{\perp}\right\rangle$ to be greater than 0. (f) Subwaves of $\delta E_{\perp 1}$ and $\delta B_{\perp 2}$ at a time period of [0.6,3] s, exhibiting their anticorrelation. (g) Subband of $\delta E_{\perp 2}$ and $\delta B_{\perp 1}$ in the same period range, exhibiting their positive correlation but with a phase lag between each other.

To further illustrate the evidence for turbulence dissipation, we calculate the scale-dependent cross-coherence (CC) and phase difference $(\varphi)$ of the different variable pairs $\left(\delta E_{\perp 1}, \delta J_{\perp 1}\right)$, $\left(\delta E_{\perp 2}, \delta J_{\perp 2}\right),\left(\delta E_{\perp 1}, \delta B_{\perp 2}\right)$, and $\left(\delta E_{\perp 2}, \delta B_{\perp 1}\right)$. The subscripts $\perp 1$ and $\perp 2$ represent the two directions perpendicular to the local mean magnetic field vector. At timescales between 0.6 and $2 \mathrm{~s}$, the high cross-coherence $\left(\mathrm{CC}\left(\delta E_{\perp 1}, \delta J_{\perp 1}\right) \sim 0.79\right.$ and $\left.\mathrm{CC}\left(\delta E_{\perp 2}, \delta J_{\perp 2}\right) \sim 0.74\right)$ and the phase difference of less than $90^{\circ}\left(\varphi\left(\delta E_{\perp 1}, \delta J_{\perp 1}\right) \sim 81^{\circ}\right.$ and $\left.\varphi\left(\delta E_{\perp 2}, \delta J_{\perp 2}\right) \sim 41^{\circ}\right)$ indicate significant dissipation in agreement with our measurement of $\langle\delta \boldsymbol{J} \cdot \delta \boldsymbol{E}\rangle$. The high cross-coherence $\left(\mathrm{CC}\left(\delta E_{\perp 1}, \delta B_{\perp 2}\right) \sim 0.89\right.$ and $\left.\mathrm{CC}\left(\delta E_{\perp 2}, \delta B_{\perp 1}\right) \sim 0.85\right)$ as well as the phase differences $\left(\varphi\left(\delta E_{\perp 1}, \delta B_{\perp 2}\right) \sim-175^{\circ}>-180^{\circ}\right.$ and $\left.\varphi\left(\delta E_{\perp 2}, \delta B_{\perp 1}\right) \sim 51^{\circ}>0^{\circ}\right)$ provide evidence that the ion cyclotron waves propagate along $B_{0}$ and dissipate. As an example, sub-intervals of $\left(\delta E_{\perp 1}, \delta J_{\perp 1}\right)$, $\left(\delta E_{\perp 2}, \delta J_{\perp 2}\right),\left(\delta E_{\perp 1}, \delta B_{\perp 2}\right)$, and $\left(\delta E_{\perp 2}, \delta B_{\perp 1}\right)$ at periods of $[0.6,2]$ $\mathrm{s}$, after wavelet decomposition from the original time sequences, are plotted in Figures 3(d)-(g).

\subsection{Scale-dependent Profile of the Energy Dissipation Rate Spectrum}

We show the probability distribution functions (PDFs) of $\varepsilon_{\mathrm{ECR}}=\frac{1}{4}\left(\delta \widetilde{\boldsymbol{J}} \cdot \delta \widetilde{\boldsymbol{E}}^{*}+\delta \widetilde{\boldsymbol{J}}^{*} \cdot \delta \widetilde{\boldsymbol{E}}\right)$ and their parallel and perpendicular components on symmetric logarithmic scales, $\quad \operatorname{PDF}\left(\lg _{\text {sym }}\left(\varepsilon_{\mathrm{ECR}, \text { trace }}\right)\right), \quad \operatorname{PDF}\left(\lg _{\text {sym }}\left(\varepsilon_{\mathrm{ECR}, \|}\right)\right), \quad$ and $\operatorname{PDF}\left(\lg _{\mathrm{sym}}\left(\varepsilon_{\mathrm{ECR}, \perp}\right)\right)$ in Figures $4(\mathrm{a})-(\mathrm{c})$. The double-peaked pattern of $\operatorname{PDF}\left(\lg _{\text {sym }}\left(\varepsilon_{\mathrm{ECR}}\right)\right)$ at different scales (see Figures 4(a)-(c)) is a characteristic of variables with alternating positive and negative values. For example, the PDF of a sinusoidal function would likewise show a doublepeaked distribution. Superposed are the ensemble-averaged profiles, $\left\langle\lg _{\mathrm{sym}}\left(\varepsilon_{\mathrm{ECR}}\right)\right\rangle$, as functions of scale. The symmetric pattern of $\operatorname{PDF}\left(\lg _{\text {sym }}\left(\varepsilon_{\mathrm{ECR}}\right)\right)$ at scales larger than $5 \mathrm{~s}$ leads to $\left\langle\lg _{\text {sym }}\left(\varepsilon_{\mathrm{ECR}}\right)\right\rangle \sim 0$. The positive bump around $1 \mathrm{~s}$ is caused by the asymmetry of $\operatorname{PDF}\left(\lg _{\text {sym }}\left(\varepsilon_{\mathrm{ECR}}\right)\right)$. The standard deviation of $\lg _{\text {sym }}\left(\varepsilon_{\mathrm{ECR}}\right), \pm \sigma\left(\lg _{\mathrm{sym}}\left(\varepsilon_{\mathrm{ECR}}\right)\right)$, plotted as horizontal error bars 

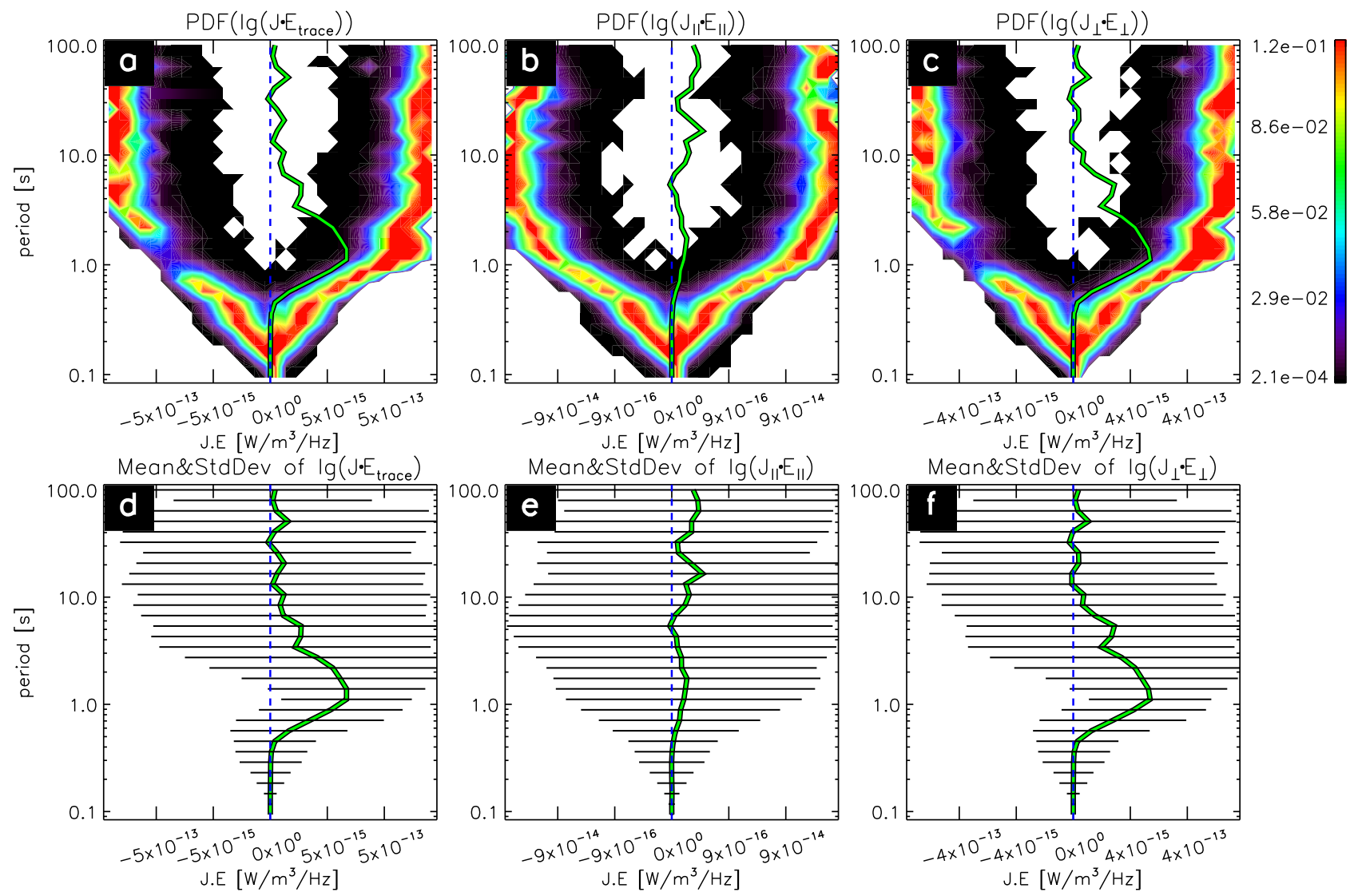

Figure 4. Scale dependence of local and global energy conversion rates in all directions, in the parallel direction, and in the perpendicular direction (left, middle, and right columns). (a) Probability distribution of $\lg _{\text {sym }}\left(\frac{1}{4}\left(\delta \widetilde{\boldsymbol{J}} \cdot \delta \widetilde{\boldsymbol{E}}^{*}+\delta \widetilde{\boldsymbol{J}}^{*} \cdot \delta \widetilde{\boldsymbol{E}}\right)\right)$ at various periods from $100 \mathrm{~s}$ down to $0.1 \mathrm{~s}$. Note that the $x$-axis is plotted in symmetric logarithmic $\left(\lg _{\text {sym }}\right)$ scale, and the probability density is shown with rainbow colors. The scale-dependent profile of the global average value, $\left\langle\lg _{\text {sym }}\left(\frac{1}{4}\left(\delta \widetilde{\boldsymbol{J}} \cdot \delta \widetilde{\boldsymbol{E}}^{*}+\delta \widetilde{\boldsymbol{J}}^{*} \cdot \boldsymbol{\delta} \widetilde{\boldsymbol{E}}\right)\right)\right\rangle$, is plotted as a green curve, which shows a positive bulge around the period of $1 \mathrm{~s}$, indicating a net energy conversion from electromagnetic fluctuations to the plasma. (b) Probability distribution of $\lg _{\text {sym }}\left(\frac{1}{4}\left(\delta \widetilde{\boldsymbol{J}}_{\|} \cdot \delta \widetilde{\boldsymbol{E}}_{\|}^{*}+\delta \widetilde{\boldsymbol{J}}_{\|}^{*} \cdot \delta \widetilde{\boldsymbol{E}}_{\|}\right)\right)$at various periods of [0.1, 100] s. The range of the $x$-axis is about 5 times smaller than that in panel (a). There is no prominent positive enhancement of $\left\langle\lg _{\text {sym }}\left(\frac{1}{4}\left(\delta \widetilde{\boldsymbol{J}}_{\|} \cdot \delta \widetilde{\boldsymbol{E}}_{\|}^{*}+\delta \widetilde{\boldsymbol{J}}_{\|}^{*} \cdot \delta \widetilde{\boldsymbol{E}}_{\|}\right)\right)\right\rangle$around 1 s. (c) Probability distribution of $\lg _{\text {sym }}\left(\frac{1}{4}\left(\boldsymbol{\delta} \widetilde{\boldsymbol{J}}_{\perp} \cdot \boldsymbol{\delta} \widetilde{\boldsymbol{E}}_{\perp}^{*}+\boldsymbol{\delta} \widetilde{\boldsymbol{J}}_{\perp}^{*} \cdot \boldsymbol{\delta} \widetilde{\boldsymbol{E}}_{\perp}\right)\right)$ from $100 \mathrm{~s}$ down to $0.1 \mathrm{~s}$. An evident positive bump of $\left\langle\lg\right.$ sym $\left.\left(\frac{1}{4}\left(\boldsymbol{\delta} \widetilde{\boldsymbol{J}}_{\perp} \cdot \boldsymbol{\delta} \widetilde{\boldsymbol{E}}_{\perp}^{*}+\delta \widetilde{\boldsymbol{J}}_{\perp}^{*} \cdot \boldsymbol{\delta} \widetilde{\boldsymbol{E}}_{\perp}\right)\right)\right\rangle$ appears at around $1 \mathrm{~s}$, indicating that the net energy conversion is mainly from fluctuations with a significant $\delta \boldsymbol{E}_{\perp}$ to the plasma particles. (d), (e), and (f) Scale-dependent global mean values and standard deviations of $\lg _{\text {sym }}\left(\frac{1}{4}\left(\boldsymbol{\delta} \widetilde{\boldsymbol{J}} \cdot \delta \widetilde{\boldsymbol{E}}^{*}+\delta \widetilde{\boldsymbol{J}}^{*} \cdot \boldsymbol{\delta} \widetilde{\boldsymbol{E}}\right)\right), \lg _{\mathrm{sym}}\left(\frac{1}{4}\left(\boldsymbol{\delta} \widetilde{\boldsymbol{J}}_{\|} \cdot \boldsymbol{\delta} \widetilde{\boldsymbol{E}}_{\|}^{*}+\delta \widetilde{\boldsymbol{J}}_{\|}^{*} \cdot \boldsymbol{\delta} \widetilde{\boldsymbol{E}}_{\|}\right)\right)$, and $\lg _{\text {sym }}\left(\frac{1}{4}\left(\boldsymbol{\delta} \widetilde{\boldsymbol{J}}_{\perp} \cdot \boldsymbol{\delta} \widetilde{\boldsymbol{E}}_{\perp}^{*}+\delta \widetilde{\boldsymbol{J}}_{\perp}^{*} \cdot \boldsymbol{\delta} \widetilde{\boldsymbol{E}}_{\perp}\right)\right)$

in Figures 4(d)-(f), reduces with decreasing scale. We note that $\left\langle\lg _{\mathrm{sym}}\left(\varepsilon_{\mathrm{ECR}}\right)\right\rangle-\sigma\left(\lg _{\mathrm{sym}}\left(\varepsilon_{\mathrm{ECR}}\right)\right)>0$ and $\left\langle\lg _{\mathrm{sym}}\left(\varepsilon_{\mathrm{ECR}}\right)\right\rangle+$ $\sigma\left(\lg _{\mathrm{sym}}\left(\varepsilon_{\mathrm{ECR}}\right)\right)>0$ for both traces, and the perpendicular component is positive around $1 \mathrm{~s}$, strongly suggesting ongoing dissipation. The maximum value of $\left\langle\lg _{\mathrm{sym}}\left(\varepsilon_{\mathrm{ECR}}\right)\right\rangle$ approaches $5 \times 10^{-14} \mathrm{~W} \mathrm{~m}^{-3} \mathrm{~Hz}^{-1}$.

The merit of this work for our understanding of turbulence is summarized in Figure 5. In Figure 5(a), energy injected at the large outer scale cascades toward smaller scales, according to the absolute equilibrium distribution of the ideal system invariant. There are two inertial ranges of plasma turbulence: the MHD inertial range and the electron inertial range in which electrons still behave approximately fluid-like. Different from the MHD inertial range, turbulence energy is dissipated in the electron inertial range due to ion kinetics. The leftover energy is then transferred to electron scales and ultimately converted to electron energization. For more details about the energy transfer, channeling and conversion, we refer readers to the review chapters in the monograph edited by Kiyani et al. (2015). Moreover, in the case of plasma instability, at some point in the plasma, part of the turbulence energy may be injected around ion scales, and then dissipated as well as cascaded to other scales.

\section{Summary and Discussion}

\subsection{Integral of the Energy Dissipation Rate Spectrum and Comparison with the Cascade Rate}

In this work, magnetosheath turbulence has been demonstrated to be an ideal laboratory to study the turbulence dissipation of collisionless cosmic plasmas with state-of-the-art measurements from the $M M S$ mission. In Figure 5(b), the trace power spectral density (PSD) of the magnetic turbulence has a conjunction with an enhancement around ion scales given by the thermal proton gyroradius and proton inertial length $\left(k \rho_{\mathrm{p}}=1, k d_{\mathrm{p}}=1\right)$, connecting the shallower and steeper power laws at MHD and sub-ion scales, respectively. The positive bump of $\left\langle\lg _{\mathrm{sym}}\left(\varepsilon_{\mathrm{ECR}}\right)\right\rangle$ is located at this conjunction, with the ion scales being located there as well. The integral 

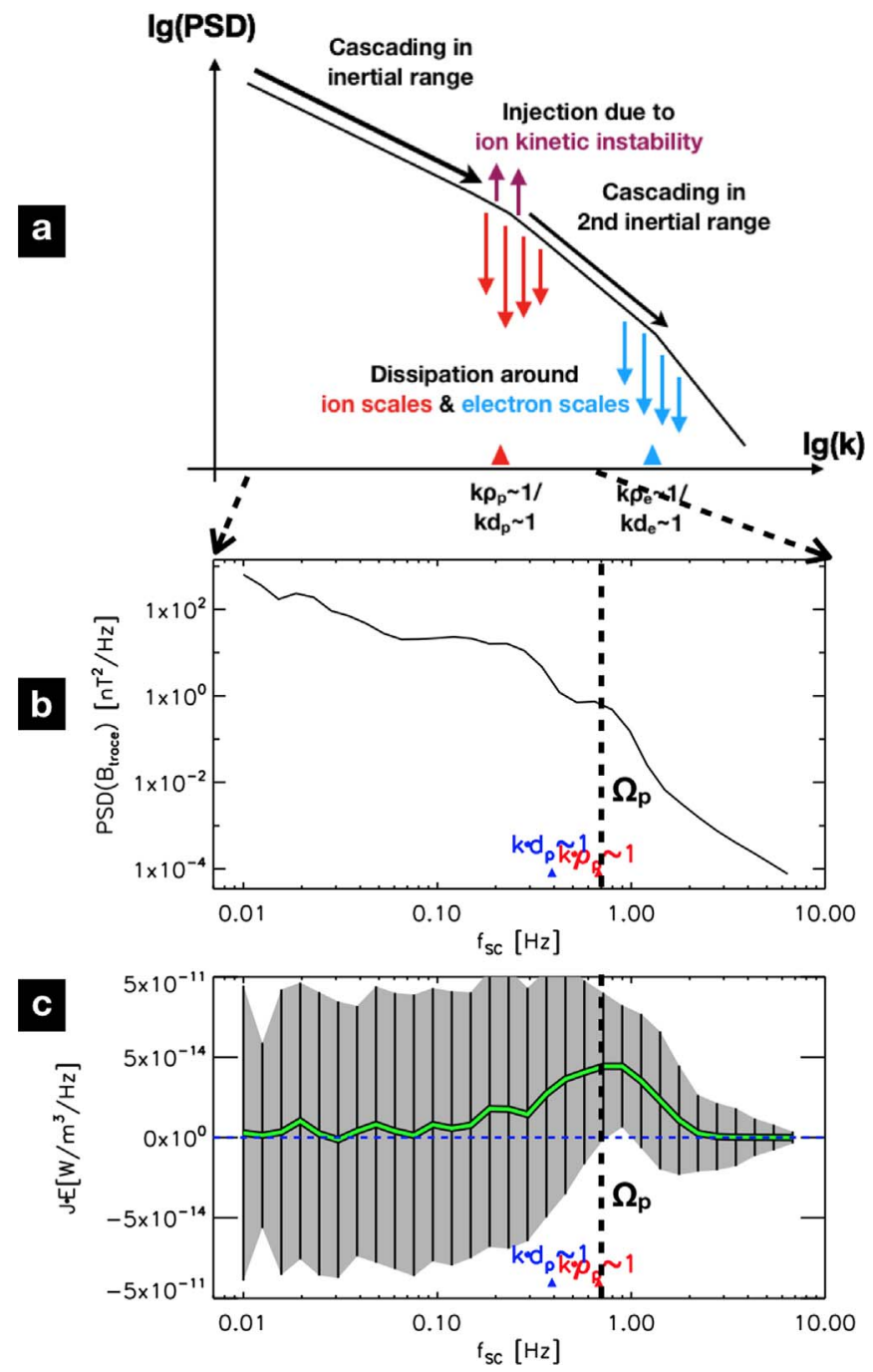

Figure 5. Scenario of turbulence cascade and dissipation, and in situ measurements of the dissipation rate spectrum around ion kinetic scales. (a) A sketch illustrating the physical processes occurring in three segments of the magnetic power spectrum. The energy cascades into the MHD inertial range and is partially (or mainly) dissipated around the ion and sub-ion scales, leaving the residual energy to cascade down to the electron scales in the second (electron) inertial range until it finally dissipates at sub-electron scales. On the other hand, due to kinetic instability, turbulence energy may be injected around particle kinetic scales. (b) Power spectral density of magnetic field turbulence, which shows a transition with some enhancements superposed on the break between the MHD regime $\left(f_{\mathrm{SC}}<0.1 \mathrm{~Hz}\right)$ and the sub-ion kinetic regime $\left(f_{\mathrm{SC}}>1 \mathrm{~Hz}\right)$. (c) Spectrum of energy conversion rate between the fluctuating field energy and plasma kinetic energy. The average and standard deviation of the energy conversion rate spectrum are plotted as the green profile and the gray area. The global average energy conversion rate spectrum starts to rise near $0.1 \mathrm{~Hz}$, approaches $5 \times 10^{-14}$ $\mathrm{W} \mathrm{m}{ }^{-3} \mathrm{~Hz}^{-1}$ near $0.8 \mathrm{~Hz}$, and then falls down beyond $1 \mathrm{~Hz}$.

$\int_{f_{\text {min,dissip }}}^{f_{\text {max,dissip }}} \varepsilon_{\mathrm{ECR}, \text { trace }} d f$, with $f_{\text {min,dissip }}=0.2 \mathrm{~Hz}$ and $f_{\text {max,dissip }}=$ $2 \mathrm{~Hz}$, is calculated to be $1.2 \times 10^{-14} \mathrm{~W} \mathrm{~m}^{-3}$ for the energy dissipation rate per unit volume, equivalent to $0.5 \times$ $10^{6} \mathrm{~W} \mathrm{~kg}^{-1}$ when considering the local number density of $15 \mathrm{~cm}^{-3}$. This value accounts for a significant fraction of the energy cascade rate in the magnetosheath turbulence (Hadid et al. 2018). The frequency range of the positive bump of $\left\langle\lg _{\text {sym }}\left(\varepsilon_{\mathrm{ECR}}\right)\right\rangle$ in the range $[0.2 \mathrm{~Hz}, 2 \mathrm{~Hz}]$ corresponds to spatial scales of $\left[1.2 d_{\mathrm{p}}, 12 d_{\mathrm{p}}\right]$. This finding is consistent with the simulation results by Yang et al. (2019), who find the $\boldsymbol{J} \cdot \boldsymbol{E}$ dissipation to be dominant around $\left[6 d_{\mathrm{p}}, 16 d_{\mathrm{p}}\right]$. The dissipation
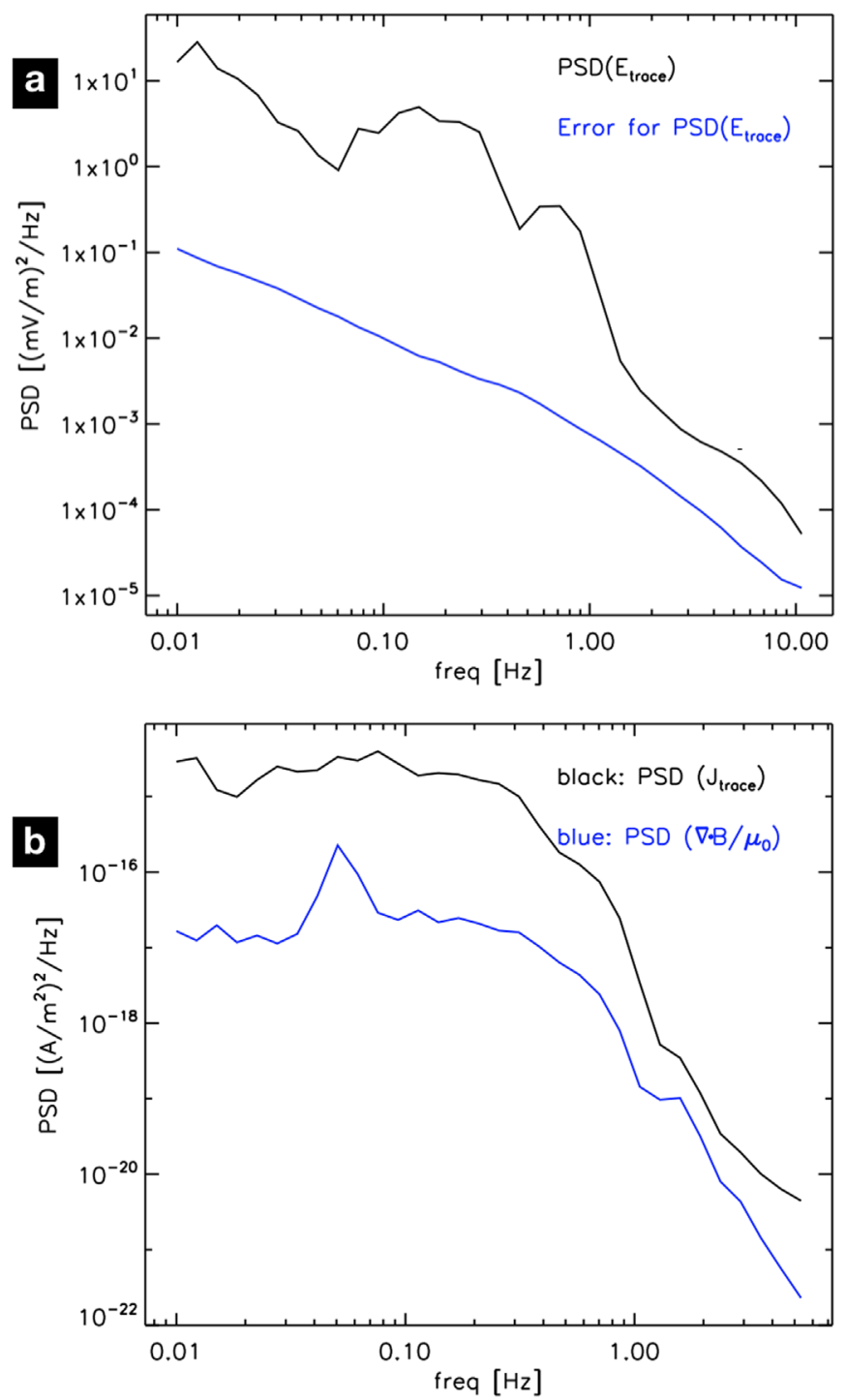

Figure 6. Reliability analysis of $\operatorname{PSD}\left(\boldsymbol{E}_{\left\langle\boldsymbol{V}_{i}\right\rangle \text { trace }}\right)$ and $\operatorname{PSD}\left(\boldsymbol{J}_{\text {trace }}\right)$. (a) $\operatorname{PSD}$ profile of $\boldsymbol{E}_{\langle\boldsymbol{V} i\rangle}$ (black curve) greater than its uncertainty estimate $\triangle \operatorname{PSD}\left(\boldsymbol{E}_{\langle\boldsymbol{V} i\rangle}\right.$,trace $)$ (blue curve), indicating that the spectral density of $\operatorname{PSD}\left(\boldsymbol{E}_{\langle\boldsymbol{V} i\rangle}\right.$,trace $)$ is credible in the frequency range from $0.01 \mathrm{~Hz}$ up to $10 \mathrm{~Hz}$. (b) PSD profile of $\boldsymbol{J}$ (black curve) well above its uncertainty estimate $\operatorname{PSD}\left(\triangle \boldsymbol{J}=\nabla \cdot \boldsymbol{B} / \mu_{0}\right)$ (blue curve), demonstrating the credibility of $\operatorname{PSD}\left(\boldsymbol{J}_{\text {trace }}\right)$ throughout the frequency range under investigation.

rate revealed here is about two orders of magnitude higher than the cascade rate in the solar wind. Ion cyclotron wave dissipation is a prevalent phenomenon in magnetosheath turbulence, which is also confirmed by the existence of more cases in our preliminary survey of the data. Furthermore, we find that the dissipation under study tends to be anisotropic with a preferential direction perpendicular to the background magnetic field vector.

\subsection{Credibility of the Power Spectral Densities of the Current Density and Electric Field}

In this subsection, we demonstrate the reliability of adopting the current density and electric field spectra to calculate the spectrum of the energy conversion rate.

It is necessary to investigate whether or not the spectrum of the energy conversion rate, $\varepsilon_{\mathrm{ECR}}=\frac{1}{4}\left(\delta \widetilde{\boldsymbol{J}} \cdot \delta \widetilde{\boldsymbol{E}}^{*}+\delta \widetilde{\boldsymbol{J}}^{*} \cdot \delta \widetilde{\boldsymbol{E}}\right)$, 

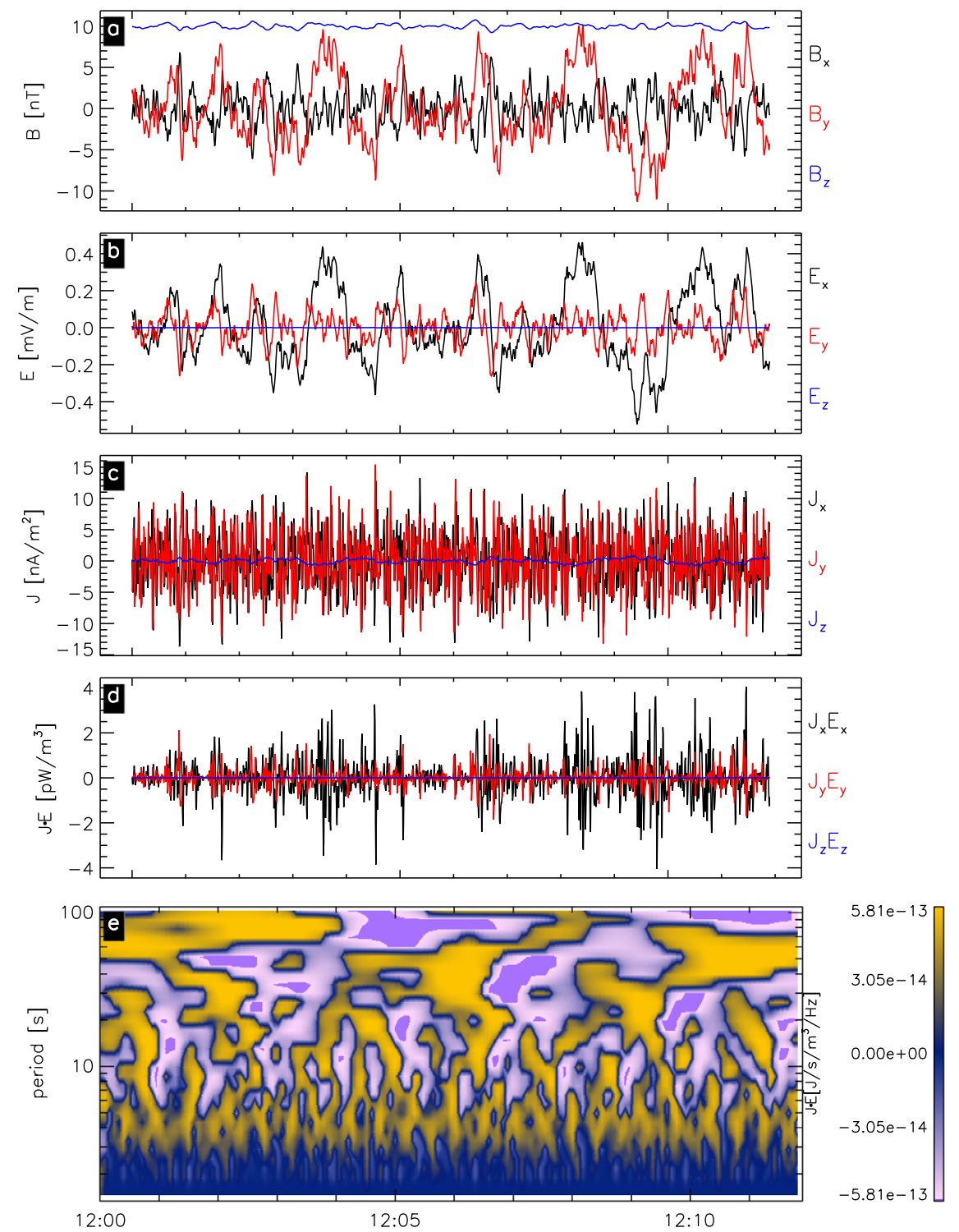

Figure 7. Synthetic turbulent fluctuations created as a superposition of Alfvén and ion cyclotron waves, and associated energy conversion characteristics. (a) and (b) Time sequences of simulated $B$ and $E$ fluctuations with good correlation between $B_{\mathrm{y}}$ and $E_{\mathrm{x}}$ and anticorrelation between $B_{\mathrm{x}}$ and $E_{\mathrm{y}}$, clearly displaying a signature of Alfvénic fluctuations propagating along the $Z$-direction. The relationship between $\boldsymbol{B}$ and $\boldsymbol{E}$ in the model is similar to the observation data shown in Figures 3(f) and (g). (c) Time sequences of simulated $\boldsymbol{J}$ fluctuations, showing oscillations at higher frequencies. (d) Oscillations of $\boldsymbol{J} \cdot \boldsymbol{E}$ around zero with alternating positive and negative values, which is similar to Figure 3 (a). (e) Time-period spectra of $\frac{1}{4}\left(\delta \widetilde{\boldsymbol{J}} \cdot \delta \widetilde{\boldsymbol{E}}^{*}+\delta \widetilde{\boldsymbol{J}}^{*} \cdot \delta \widetilde{\boldsymbol{E}}\right)$, representing local pseudo-energy conversion rate spectra between electromagnetic and plasma energies. The full-time golden patch at periods of $[2,6] \mathrm{s}$ displays prevalent positive values, demonstrating the net dissipation of electromagnetic energy, which is hidden in the time sequence but uncovered in the time-period spectrogram.

is reliable throughout the frequency range of interest. To achieve this goal, we first test the reliability of PSDs for $\boldsymbol{J}$ and $\boldsymbol{E}_{\left\langle V_{i}\right\rangle}$, the observations of which are required to be higher than the noise level. The error estimate of $\boldsymbol{J}, \Delta \boldsymbol{J}$, is taken as $\langle\nabla \cdot \boldsymbol{B}\rangle_{\mathrm{vol}} / \mu_{0}$, the divergence of $\boldsymbol{B}$ divided by $\mu_{0}$ as obtained with the curlometer method (Dunlop et al. 1988). The uncertainty of $\operatorname{PSD}(\boldsymbol{J}), \triangle \operatorname{PSD}(\boldsymbol{J})$, can therefore be approximated as $\operatorname{PSD}(\triangle \boldsymbol{J})$. The uncertainty of $\operatorname{PSD}(\boldsymbol{E}), \triangle \operatorname{PSD}(\boldsymbol{E})$, is derived based on the error of the second-order structure function. The second-order structure function is the ensemble average of the squared difference,

$$
\begin{aligned}
\operatorname{SF}(E ; \tau) & =\left\langle|E(t+\tau)-E(t)|^{2}\right\rangle=\left\langle|d E(t)|^{2}\right\rangle \\
& =\frac{1}{N} \sum_{i=1}^{N}\left|d E_{i}\right|^{2},
\end{aligned}
$$

where $\tau$ is the time lag for the variable difference. Following the work of Wicks et al. (2011), the squared error of the structure function $\Delta \mathrm{SF}^{2}$ can be expressed as a function of the nominal error of the variable $\Delta E$ and the structure function itself:

$$
\begin{aligned}
\Delta \mathrm{SF}^{2} & =\sum_{i=1}^{N}\left|\frac{\partial \mathrm{SF}}{\partial d E_{i}}\right|^{2}\left(\Delta d E_{i}\right)^{2} \\
& =(\triangle d E)^{2} \cdot \sum_{i=1}^{N}\left(\frac{2}{N}\right)^{2} d E_{i}^{2}=(\triangle d E)^{2} \cdot \frac{4}{N} \cdot \mathrm{SF} \\
& =\frac{8}{N} \cdot \mathrm{SF} \cdot(\triangle E)^{2},
\end{aligned}
$$

where the squared difference error $(\triangle d E)^{2}$ is simplified to be twice the squared variable $(\triangle E)^{2}$, assuming the independence 

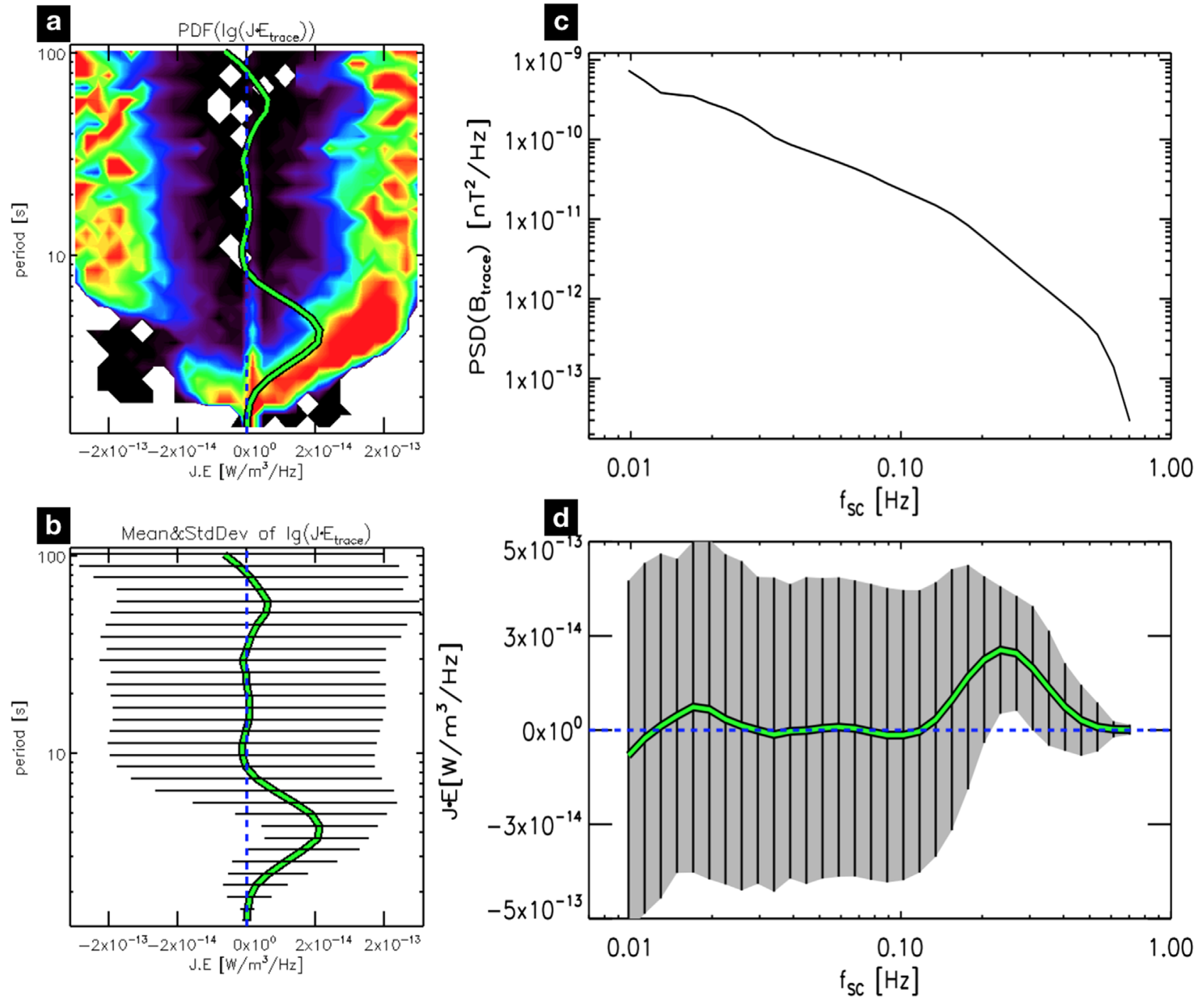

Figure 8. Energy conversion rate spectrum derived from modeling synthetic spacecraft data illustrating the net energy dissipation rate at ion kinetic scales. (a) Rainbow color-coded probability density function of $\lg _{\text {sym }}\left(\frac{1}{4}\left(\delta \widetilde{\boldsymbol{J}} \cdot \delta \widetilde{\boldsymbol{E}}^{*}+\delta \widetilde{\boldsymbol{J}}^{*} \cdot \delta \widetilde{\boldsymbol{E}}\right)\right)$ at periods from $100 \mathrm{~s}$ down to $1 \mathrm{~s}$, superposed by the green-colored scaledependent profile of the global average value, $\left\langle\lg _{\text {sym }}\left(\frac{1}{4}\left(\delta \widetilde{\boldsymbol{J}} \cdot \delta \widetilde{\boldsymbol{E}}^{*}+\delta \widetilde{\boldsymbol{J}}^{*} \cdot \delta \widetilde{\boldsymbol{E}}\right)\right)\right\rangle$. The positive bump at periods below $10 \mathrm{~s}$ indicates a net energy dissipation of electromagnetic turbulence. (b) Scale-dependent global mean values (green line) and standard deviations (black horizontal bars) of $\lg _{\text {sym }}\left(\frac{1}{4}\left(\delta \widetilde{\boldsymbol{J}} \cdot \delta \widetilde{\boldsymbol{E}}^{*}+\delta \widetilde{\boldsymbol{J}}^{*} \cdot \delta \widetilde{\boldsymbol{E}}\right)\right)$ (c) Trace power spectrum density of synthetic magnetic field fluctuations. The spectral break around $0.2 \mathrm{~Hz}$ and the exponential drop at the high-frequency end are defined in our model. (d) Same as panel (b), but rotated and replotted in the frequency domain.

of variables at different times. The relation between the uncertainties of $\triangle \mathrm{PSD}$ and $\triangle \mathrm{SF}$ is derived to be

$$
\triangle \mathrm{PSD}^{2}=\left|\frac{\partial \mathrm{PSD}}{\partial \mathrm{SF}}\right|^{2} \cdot \Delta \mathrm{SF}^{2} \sim \alpha^{2} f^{-2} \cdot \Delta \mathrm{SF}^{2},
$$

where the power-law function of the PSD can be approximated with its corresponding structure function as

$$
\operatorname{PSD}(f) \sim \alpha f^{-1} \cdot \operatorname{SF}(f) .
$$

We plot the resulting uncertainties, $\triangle \operatorname{PSD}\left(J_{\text {trace }}\right)$ and $\triangle \operatorname{PSD}\left(E_{\left\langle V_{i}\right\rangle, \text { trace }}\right)$, in Figure 6. We find that $\triangle \operatorname{PSD}\left(J_{\text {trace }}\right)<$ $\operatorname{PSD}\left(J_{\text {trace }}\right)$ and $\triangle \operatorname{PSD}\left(E_{\langle V i\rangle \text { trace }}\right)<\operatorname{PSD}\left(E_{\langle V i\rangle \text { trace }}\right)$ in the frequency range of $[0.01,10] \mathrm{Hz}$, clearly indicating the reliability of the spectra for $\boldsymbol{J}$ and $\boldsymbol{E}_{\left\langle\boldsymbol{V}_{i}\right\rangle}$ as well as the energy conversion rate $\varepsilon_{\mathrm{ECR}}$.

\subsection{Dissipation Rate Spectrum as Derived from Modeled Synthetic Spacecraft Data}

To highlight the scientific significance of the observed energy conversion rate spectrum, we analyze artificially sampled fluctuations as constructed from a synthetic model composed of Alfvén waves at MHD and kinetic scales, and then calculate the corresponding energy conversion rate spectrum (see Figures 7 and 8).

The characteristic signatures indicating the dissipation as illustrated in Figures 3(b), 4, and 5 are confirmed in the modeled synthetic spacecraft data. We set up a model spectrum by superposing a package of linear wave modes (Alfvén waves and ion cyclotron waves) with random phases. Their magnetic amplitudes are set to the typically observed power spectral profile. The other variables, e.g., electric field and current density, are obtained according to their polarization and 
magnetic field fluctuations in linear Vlasov-Maxwell theory with our NHDS code (Verscharen \& Chandran 2018). This defines the spatial fields of $(\delta \boldsymbol{B}, \delta \boldsymbol{E}, \delta \boldsymbol{J})$ and their time evolution Afterwards, a virtual spacecraft crosses the time-evolving spatial fields and samples the variables. Finally, time sequences of $(\delta \boldsymbol{B}, \delta \boldsymbol{E}, \delta \boldsymbol{J})$ are acquired from the virtual measurements. The method proposed in this work is then applied to the time sequences of $(\delta \boldsymbol{B}, \delta \boldsymbol{E}, \delta \boldsymbol{J})$. As in the real observations, we see the highly oscillating pattern of the $\delta \boldsymbol{J}$ sequence, which is a signal of the superposed waves rather than noise (see Figure 7(c)). Likewise, we extract the physics of energy conversion from the oscillating time sequence of $\delta \boldsymbol{J} \cdot \delta \boldsymbol{E}$ in Figure $7(\mathrm{~d})$. The real physics of turbulence energy dissipation lies in the time-period spectra of $\frac{1}{4}\left(\delta \widetilde{\boldsymbol{J}} \cdot \delta \widetilde{\boldsymbol{E}}^{*}+\delta \widetilde{\boldsymbol{J}}^{*} \cdot \delta \widetilde{\boldsymbol{E}}\right)$ (Figure $7(\mathrm{e})$ ) and the probability density function of $\lg _{\text {sym }}\left(\frac{1}{4}\left(\delta \widetilde{\boldsymbol{J}} \cdot \delta \widetilde{\boldsymbol{E}}^{*}+\delta \widetilde{\boldsymbol{J}}^{*} \cdot \delta \widetilde{\boldsymbol{E}}\right)\right)$ (Figures $8(\mathrm{a})$ and (b)). The analysis results from the modeled synthetic spacecraft data strongly suggest that the turbulence dissipation rate spectrum revealed by $M M S$ data is credible and valuable.

\subsection{Prospects Based on and beyond Measuring the Energy Conversion Rate Spectrum}

Our approach will allow us to study the dissipation of other wave modes and intermittent coherent structures in space plasmas in the future. It is also capable of finding evidence for wave excitation due to local instability. Another important issue is the investigation of the source of the dissipated energy, which is beyond the scope of this work and needs to be addressed in the future. There are three possibilities for the energy source: (1) energy cascaded from MHD scales down to kinetic scales, (2) spatial accumulation of energy due to the convergence of Poynting flux, and (3) wave growth due to local instability happening elsewhere. Although this work opens a new perspective for understanding the dissipation of turbulent electromagnetic energy, it does not describe the ultimate energy deposit from oscillating bulk kinetic energy to thermal kinetic energy, which needs another way and different tools to be diagnosed (Yang et al. 2017).

The authors are grateful to the teams of the $M M S$ spacecraft for providing the data. The authors from China are supported by the National Natural Science Foundation of China (NSFC) under contracts 41574168, 41874200, 41421003, 41874199, 41474147, and 41674171. J.S.H. is also supported by the National Young Talent Program of China. D.V. is supported by the STFC Ernest Rutherford Fellowship ST/P003826/1. J.-S. $\mathrm{H}$. proposed the idea, analyzed the data, wrote the manuscript, and led the discussions. D.D, T.Y.W., and X.Y.Z. analyzed part of the data. D.V. contributed the eigenmode data from the linear kinetic theory. Y.K., G.L., and J.B. contributed to the development and operation of payloads on board $M M S$ and the interpretation of data from $M M S$. All authors discussed the results and improved the manuscript.

\section{ORCID iDs}

Jiansen He (ib https://orcid.org/0000-0001-8179-417X Tieyan Wang (iD https:// orcid.org/0000-0003-3072-6139 Xingyu Zhu (iD https://orcid.org/0000-0002-1541-6397 Daniel Verscharen (iD https://orcid.org/0000-0002-0497-1096 Chuanyi Tu (ib https://orcid.org/0000-0002-9571-6911 Yuri Khotyaintsev (iD https://orcid.org/0000-0001-5550-3113 Guan Le (i) https://orcid.org/0000-0002-9504-5214

\section{References}

Anderson, B. J., Fuselier, S. A., \& Murr, D. 1991, GeoRL, 18, 1955

Bale, S. D., Kellogg, P. J., Mozer, F. S., Horbury, T. S., \& Reme, H. 2005, PhRvL, 94, 215002

Banerjee, S., \& Galtier, S. 2013, PhRvE, 87, 013019

Biskamp, D. 2003, Magnetohydrodynamic Turbulence (Cambridge: Cambridge Univ. Press)

Bruno, R., \& Carbone, V. 2013, LRSP, 10, 2

Bruno, R., \& Trenchi, L. 2014, ApJL, 787, L24

Burch, J. L., Torbert, R. B., Phan, T. D., et al. 2016, Sci, 352, aaf2939

Chandran, B. D., Li, B., Rogers, B. N., Quataert, E., \& Germaschewski, K. 2010, ApJ, 720, 503

Chasapis, A., Yang, Y., Matthaeus, W. H., et al. 2018, ApJ, 862, 32

Chen, C. H. K., \& Boldyrev, S. 2017, ApJ, 842, 122

Chen, C. H. K., Klein, K. G., \& Howes, G. G. 2019, NatCo, 10, 740

Duan, D., He, J., Pei, Z., et al. 2018, ApJ, 865, 89

Dunlop, M. W., Southwood, D. J., Glassmeier, K. H., \& Neubauer, F. M. 1988, AdSpR, 8, 273

Frisch, U. 1995, Turbulence: the Legacy of AN Kolmogorov (Cambridge: Cambridge Univ. Press)

Fu, H., Vaivads, A., Khotyaintsev, Y. V., et al. 2017, GeoRL, 44, 37

Gary, S. P., Fuselier, S. A., \& Anderson, B. J. 1993, JGRA, 98, 1481

Gary, S. P., \& Smith, C. W. 2009, JGRA, 114, A12105

Hadid, L. Z., Sahraoui, F., Galtier, S., \& Huang, S.-Y. 2018, PhRvL, 120, 055102

He, J. S., Marsch, E., Tu, C. Y., Yao, S., \& Tian, H. 2011, ApJ, 731, 85

He, J., Wang, L., Tu, C., Marsch, E., \& Zong, Q. 2015, ApJL, 800, L31

Howes, G. G. 2015, RSPTA, 373, 20140145

Howes, G. G., Klein, K. G., \& Li, T. C. 2017, JPlPh, 83, 705830102

Howes, G. G., McCubbin, A. J., \& Klein, K. G. 2018, JPIPh, 84, 905840105

Karimabadi, H., Roytershteyn, V., Wan, M., et al. 2013, PhPl, 20, 012303

Kiyani, K. H., Chapman, S. C., Khotyaintsev, Y. V., Dunlop, M. W., \& Sahraoui, F. 2009, PhRvL, 103, 075006

Kiyani, K. H., Osman, K. T., \& Chapman, S. C. 2015, RSPTA, 373, 20140155

Klein, K. G., \& Howes, G. G. 2016, ApJL, 826, L30

Klein, K. G., Howes, G. G., \& TenBarge, J. M. 2017, JPIPh, 83, 535830401

Kolmogorov, A. N. 1941, Dokl. Akad. Nauk SSSR, 32, 16

Leamon, R. J., Matthaeus, W. H., Smith, C. W., \& Wong, H. K. 1998, ApJL, 507, L181

Matteini, L., Alexandrova, O., Chen, C. H. K., \& Lacombe, C. 2016, MNRAS, 466,945

Monin, A. S., \& Yaglom, A. M. 1975, Statistical Fluid Mechanics: Mechanics of Turbulence, Vol. 2 (Cambridge, MA: MIT Press)

Narita, Y., Glassmeier, K. H., Sahraoui, F., \& Goldstein, M. L. 2010, PhRvL, 104, 171101

Osman, K., Kiyani, K., Matthaeus, W., et al. 2015, ApJ, 815, L24

Osman, K. T., Wan, M., Matthaeus, W. H., Weygand, J. M., \& Dasso, S. 2011, PhRvL, 107, 165001

Politano, H., \& Pouquet, A. 1998, PhRvE, 57, R21

Pollock, C., Moore, T., Jacques, T. A., et al. 2016, SSRv, 199, 331

Ruan, W., He, J., Zhang, L., et al. 2016, ApJ, 825, 58

Sahraoui, F., Goldstein, M. L., Belmont, G., Canu, P., \& Rezeau, L. 2010, PhRvL, 105, 131101

Santolík, O., Parrot, M., \& Lefeuvre, F. 2003, RaSc, 38, 1010

Scannapieco, E., \& Brüggen, M. 2008, ApJ, 686, 927

Sitnov, M., Merkin, V., Roytershteyn, V., \& Swisdak, M. 2018, GeoRL, 45,4639

Sorriso-Valvo, L., Carbone, V., Veltri, P., Consolini, G., \& Bruno, R. 1999, GeoRL, 26, 1801

Torbert, R. B., Russell, C. T., Magnes, W., et al. 2016, SSRv, 199, 105

Tu, C. Y., \& Marsch, E. 1995, SSRv, 73, 1

Verscharen, D., \& Chandran, B. D. G. 2018, RNAAS, 2, 13

Wan, M., Matthaeus, W. H., Roytershteyn, V., et al. 2015, PhRvL, 114, 175002

Wang, X., Tu, C. Y., He, J. S., Marsch, E., \& Wang, L. H. 2013, ApJL, 772, L14

Wang, X., Tu, C.-Y., He, J.-S., \& Wang, L.-H. 2018, JGRA, 123, 68

Wicks, R. T., Horbury, T. S., Chen, C. H. K., \& Schekochihin, A. A. 2011, PhRvL, 106, 045001

Woodham, L. D., Wicks, R. T., Verscharen, D., \& Owen, C. J. 2018, ApJ, 856,49

Yang, Y., Matthaeus, W. H., Parashar, T. N., et al. 2017, PhRvE, 95, 061201

Yang, Y., Wan, M., Matthaeus, W. H., et al. 2019, MNRAS, 482, 4933

Zenitani, S., Hesse, M., Klimas, A., \& Kuznetsova, M. 2011, PhRvL, 106, 195003 